\title{
Taking stock of superluminous supernovae and long gamma-ray burst host galaxy comparison using a complete sample of LGRBs
}

\author{
J. Japelj ${ }^{1}$, S. D. Vergani ${ }^{2,3}$, R. Salvaterra ${ }^{4}$, L. K. Hunt ${ }^{5}$, and F. Mannucci ${ }^{5}$
}

\author{
1 INAF-Osservatorio Astronomico di Trieste, via G. B. Tiepolo 11, 34131 Trieste, Italy \\ e-mail: japelj@oats.inaf.it \\ 2 GEPI, Observatoire de Paris, PSL Research University, CNRS, Univ. Paris Diderot, Sorbonne Paris Cité, Place Jules Janssen, \\ 92195 Meudon, France \\ 3 INAF-Osservatorio Astronomico di Brera, via E. Bianchi 46, 23807 Merate, Italy \\ ${ }^{4}$ INAF-IASF Milano, via E. Bassini 15, 20133 Milano, Italy \\ 5 INAF-Osservatorio Astrofisico di Arcetri, Largo E. Fermi 5, 50125 Firenze, Italy
}

Received 29 March 2016 / Accepted 4 July 2016

\begin{abstract}
Long gamma-ray bursts (LGRBs) and superluminous supernovae (SLSNe) are both explosive transients with very massive progenitor stars. Clues about the nature of the progenitors can be found by investigating environments in which such transients occur. While studies of LGRB host galaxies have a long history, dedicated observational campaigns have only recently resulted in a high enough number of photometrically and spectroscopically observed SLSN hosts to allow statistically significant analysis of their properties. In this paper we make a comparison of the host galaxies of hydrogen-poor (H-poor) SLSNe and the Swift/BAT6 sample of LGRBs. In contrast to previous studies, we use a complete sample of LGRBs and we pay special attention to the comparison methodology and the selection of SLSN sample whose data have been compiled from the available literature. At intermediate redshifts $(0.3<z<0.7)$ the two classes of transients select galaxies whose properties (stellar mass, luminosity, star formation rate, specific star formation rate and metallicity) do not differ significantly. Moreover, the host galaxies of both classes of objects follow the fundamental metallicity relation and the fundamental plane of metallicity. In contrast to previous studies we show that at intermediate redshifts the emission line equivalent widths of the two populations are essentially the same and that the previous claims regarding the higher fraction of SLSN hosts among the extreme emission line galaxies with respect to LGRBs are mostly due to a larger fraction of strong-line emitters among SLSN hosts at $z<0.3$, where samples of LGRB hosts are small and poorly defined.
\end{abstract}

Key words. galaxies: star formation - supernovae: general - galaxies: starburst

\section{Introduction}

Distinctly more luminous than ordinary supernovae, the recently established class of superluminous supernovae (SLSN; Quimby et al. 2011) is associated with the deaths of very massive stars (e.g. Gal-Yam et al. 2009) and is being recognized as a new class of cosmic beacons that pinpoints distant star-forming galaxies (Lunnan et al. 2014; Leloudas et al. 2015; Angus et al. 2016; Perley et al. 2016b, hereafter L14, L15, A16, and P16) and lights up their environment (Berger et al. 2012; Vreeswijk et al. 2014). Owing to their extreme luminosities, together with peculiar spectral and light curve properties, understanding the nature of the progenitors and emission mechanisms involved in SLSNe has proved to be rather challenging.

Superluminous supernovae come in many flavours, which can be grouped into two subclasses based on the presence of hydrogen in their spectra (Gal-Yam 2012): hydrogen-rich SLSN-II and hydrogen-poor (H-poor) SLSN-I. The extreme luminosities of the former are commonly explained as an interaction of supernova ejecta with a dense interstellar medium (e.g. Moriya et al. 2013, but see Inserra et al. 2016). However, the physical nature of the SLSN-I type remains debated. A subclass of slowly evolving SLSN-I was proposed to be powered by radioactive decay of ${ }^{56} \mathrm{Ni}$ (Gal-Yam et al. 2009; Gal-Yam 2012). The amount of ${ }^{56} \mathrm{Ni}$ required in this case should be large, but could be produced by a pair-instability SN (Barkat et al. 1967). A SN of this nature is expected to arise in the case of a very massive star $\left(\sim 140-260 M_{\odot}\right)$ where the cascading conversion of starsupporting photons to electron-positron pairs is followed by a rapid contraction and thermonuclear explosion. On the other hand, SLSN-I emission could be powered by a central engine through the reheating of the SN ejecta by an accretion onto a central black hole (Dexter \& Kasen 2013) or spin-down of a rapidly rotating neutron star with strong magnetic fields (i.e. the magnetar model; Woosley 2010; Dessart et al. 2012; Inserra et al. 2013; Nicholl et al. 2013, 2015).

In addition to studying SLSN light curves and spectra, clues about the progenitors of SLSN can be found by investigating the environments in which they occur. SLSN host galaxies are often characterized by strong nebular emission lines (L14; L15; P16). In cases where the host is experiencing a recent and young starburst, the equivalent widths of the emission lines can give a meaningful constraint on the age of the stellar population and therefore the age and mass of a progenitor star (Thöne et al. 2015; but see Chen et al. 2015). Properties of the environment can also provide clues to distinguish between more or less probable progenitor models. For example, progenitor stars are expected to be of low metallicity in order to produce the rapidly rotating neutron star (Woosley \& Heger 2006; Yoon et al. 2006) or pair-instability SN (Langer et al. 2007) and therefore their host environment is expected to be metal poor. Studies performed so 
far have shown that the H-poor SLSNe are typically found in host galaxies of low luminosity, low stellar mass $\left(M_{\star}\right)$, low star formation rates (SFRs), and low nebular metallicities (Neill et al. 2011; Chen et al. 2013, L14; L15; A16; P16). On the other hand, host galaxies of SLSN-II type are found in galaxies spanning a wider range in luminosity, mass, and SFR.

To put the results into perspective, SLSN hosts have been compared to the host galaxies of other types of explosive transients with massive progenitors. Of particular interest is a comparison with long duration gamma-ray bursts (LGRBs; e.g. Kumar \& Zhang 2015), whose optical afterglow luminosity shortly after the explosion can vastly exceed that of SLSNe (e.g. Bloom et al. 2009), and whose progenitors are likewise being sought in their host galaxy environment (e.g. Le Floc'h et al. 2003; Savaglio et al. 2009; Levesque et al. 2010a; Graham \& Fruchter 2013; Krühler et al. 2015; Vergani et al. 2015; Perley et al. 2016c; Japelj et al. 2016). In addition to the collapsar scenario (e.g. Woosley 1993) the magnetar has also been invoked as a possible central engine of LGRBs and their accompanying supernovae (Usov 1992; Metzger et al. 2015; Greiner et al. 2015; Cano et al. 2016). If the central engine for the two transients is indeed the same, it is expected that SLSN and LGRB are preferentially found in the same environments (though the opposite is not necessarily true). Comparing the host galaxies of SLSN-I and LGRBs, L14 finds them to be similar in $M_{\star}$, SFR, specific SFR (SFR divided by stellar mass), and metallicity, especially if excluding the events at the lowest redshifts. On the contrary, spectroscopic study of L15 and photometric study of A16 conclude that the LGRB hosts are found in less extreme environments (e.g. higher $M_{\star}$ and SFRs and lower emission line EWs) than SLSN-I.

The reason for different conclusions could at least partly be attributed to the methodology and biased samples of both SLSNe and LGRBs used in the comparison. We therefore aim to take stock of this issue by comparing the properties of the host galaxies of the Swift/BAT6 complete sample of LGRBs (Salvaterra et al. 2012) and a sample of SLSN host galaxies carefully selected from the literature. We discuss the effects the selection of the SLSN sample has on our conclusions and emphasize the importance of a redshift interval assumed in the comparison of the two galaxy populations. Because of the limited statistics for the H-rich class of SLSNe (SLSN-II), our discussion is limited to the host galaxies of H-poor SLSNe.

All errors are reported at $1 \sigma$ confidence unless stated otherwise. We use a standard cosmology (Planck Collaboration XVI 2014): $\Omega_{\mathrm{m}}=0.315, \Omega_{\Lambda}=0.685$, and $H_{0}=67.3 \mathrm{~km} \mathrm{~s}^{-1} \mathrm{Mpc}^{-1}$ All quantities are computed with respect to the Chabrier initial mass function (Chabrier 2003).

\section{Data}

We used the LGRB host galaxy sample based on the complete Swift/BAT6 sample of LGRBs (Salvaterra et al. 2012). The selection of this sample of LGRBs is based on the prompt emission alone. In principle this does not introduce any bias on the host galaxy properties. Indeed, no correlations have been found to date between the prompt emission properties and those of the LGRB host galaxies (Levesque et al. 2010b; Japelj et al. 2016). The stellar masses, absolute $M_{\mathrm{B}}$ magnitudes, SFR, and metallicity of the host galaxies are published for the $0.3<z<1.0^{1}$ part of the sample (Vergani et al. 2015; Japelj et al. 2016).

1 There is also an object (GRB 060614) at $z<0.3$. As its nature is debated, we exclude it from this analysis.
From the SLSN side, we focus our study on hydrogen-poor SLSNe, in which we include both the SLSN-I and SLSN-R type events, in the same redshift range of the GRB sample $(0.3<z<1.0)$. After a close inspection of the data sets from the samples of L14, L15, A16, and P16, we noticed that the stellar masses of the overlapping subsamples differ, in some cases by an order of magnitude (where necessary, we transformed the masses to the Chabrier IMF calibration for a valid comparison). Stellar masses in all four works were obtained by modelling the photometric spectral energy distributions (SEDs) of the galaxies with synthetic models. Such high discrepancy is worrisome and therefore our selection of the sample was based principally on the criterion of the quality of stellar mass determination. We therefore discard the stellar masses from L15 as the only reference to their photometry and SED analysis is from a yet unpublished work; we also discard the stellar masses determined from SED without rest-frame near-infrared (NIR) observations. Indeed, we noticed a better agreement for the cases in which the stellar masses of the different works in the literature were determined from SED including NIR observations. It turned out that all published hosts in a redshift interval $0.3<z<0.7$ have available NIR observations; therefore, we decided to use this redshift interval (both for LGRB and SLSN samples) for the comparison to avoid introducing any additional bias due to the requirement of NIR observations when comparing average galaxy properties in Sect. 3.1. Our SLSN host galaxy sample is comprised of all the objects $(11)$ in the redshift interval $0.3<z<0.7$ presented in L14, A16, and P16. The sample is summarized in Table A.1. Based on the availability, stellar masses were adopted from the literature in the following order of preference reflecting the quality of the SEDs: P16, A16, and L14. Spectroscopic observations are available for all the galaxies in the sample.

To measure SFRs and metallicities of the part of SLSN host galaxies coming from L14 and A16 only, we used published flux densities of detected host emission lines and analysed them by following the same prescriptions as used by Japelj et al. (2016) for the LGRB host galaxies. Star formation rates were measured from extinction-corrected spectral emission lines. The lines we used as SFR tracers were (in the order of preference and based on the availability): $\mathrm{H} \alpha, \mathrm{H} \beta$ (Kennicutt 1998), and [O II] (Krühler et al. 2015). Metallicities were computed by minimizing all metallicity calibrations available for an emission-line set of each host galaxy (Maiolino et al. 2008). For the part of SLSN host galaxies coming from P16, we used the values in the paper as they comply to the same prescriptions used for the LGRB host galaxy spectra. Absolute $B$-band magnitudes of the SLSN hosts were taken from L14. For the P16 hosts, $M_{\mathrm{B}}$ were either extrapolated from their SEDs or from the available photometry in their Table 2.

\section{Results}

\subsection{Cumulative properties in the $0.3<z<0.7$ range}

For the comparison of most of the host galaxy properties it is fundamental to restrict the analysis to similar redshift ranges in order to avoid introducing systematic differences due to galaxy evolution with redshift. Therefore, for the cumulative distributions we consider only a redshift range common to our LGRB and SLSN sample of host galaxies, i.e. $0.3<z<0.7$. Mean redshifts of the SLSN (11 hosts) and LGRB (8 hosts) samples are $\langle z\rangle=0.46$ and 0.53 , respectively.

In Fig. 1 we compare cumulative distributions of stellar masses and absolute magnitudes of the two populations. Both the 

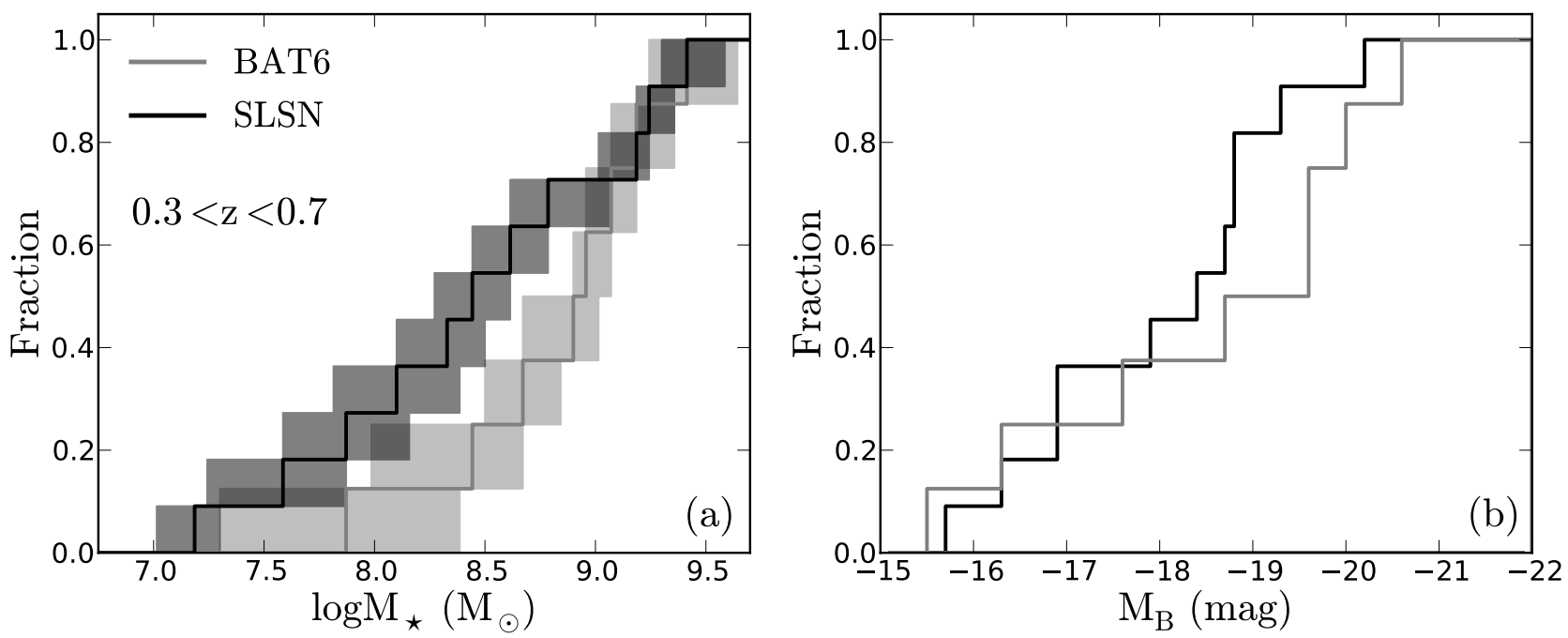

Fig. 1. Cumulative distributions of a) stellar mass and b) absolute $B$-band magnitude of SLSN and LGRB samples in the $0.3<z<0.7$ redshift range. For stellar masses the distributions were obtained via MC simulation taking into account the errors of individual measurements.
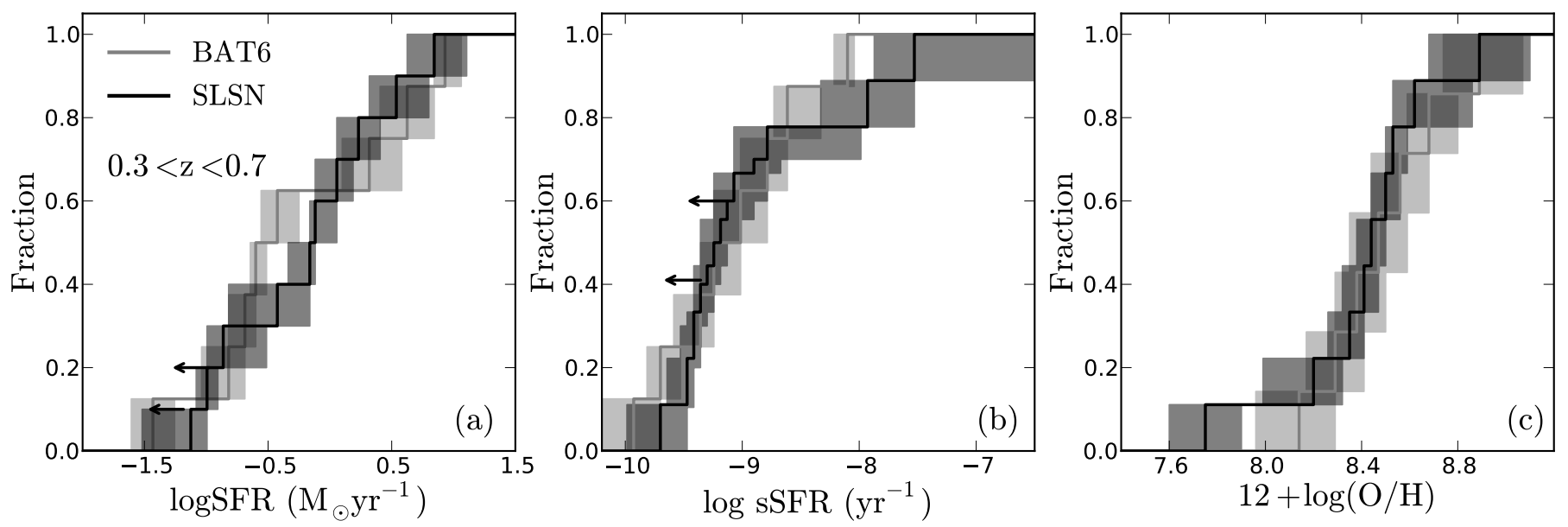

Fig. 2. Cumulative distributions of a) star formation rate; b) specific SFR; and c) metallicities of SLSN and LGRB samples in the $0.3<z<0.7$ redshift range.

number of SLSN and LGRB hosts in the samples is small; therefore, we took the errors into account (by performing MC simulation), which is shown by shaded regions in the plots (except for the $M_{B}$ values which in the literature are reported without errors). According to the Kolmogorov-Smirnov test, the probability that the two samples are drawn from the same distribution is $p_{\mathrm{KS}} \sim 0.15$ and $p_{\mathrm{KS}} \sim 0.32$ for masses and luminosities, respectively. In both cases we cannot discard the hypothesis that the two samples are drawn from the same distribution.

The SLSN host galaxy sample can be affected by selection biases (see the relevant discussion in L14 and P16). There are two factors affecting the selection of SLSN that can result in a bias of the host galaxies against dusty, massive galaxies. First, the SLSN are optically selected and therefore more difficult to detect in dusty galaxies. Second, the spectroscopic follow-up of SLSN candidates is preferentially carried out for the events significantly brighter than their hosts (see L14). To verify the robustness of our findings, we therefore artificially added massive hosts of $\log M_{\star} / M_{\odot}=11$ to the SLSN sample and we determined how many of them are needed to discard the hypothesis that the GRB and SLSN host populations are similar. We find that to reach that result (i.e. $p_{\mathrm{KS}} \lesssim 0.01$ ) we should add $\sim 15$ massive galaxies to the sample. It is hard to believe that more than half of the SLSN would be lost owing to their origin in such massive galaxies (see also discussion in P16); therefore, we conclude that this does not affect our conclusion. The conditions we are imposing, i.e., selecting our SLSN sample from those published in the literature, do not introduce any further bias. In any case, we repeated the test, this time adding low-mass hosts $\left(\log M_{\star} / M_{\odot}=7\right)$, and we find that the SLSN distribution differed significantly from that of LGRBs when $\sim 10$ low-mass host galaxies are added to the sample. Our results on the stellar masses and luminosities, therefore, do not support the hypothesis that SLSN and GRB host galaxies belong to two different populations. Finally, the uncertainty regions in the plots take only measurement errors into account. To understand the impact of small sample sizes on our conclusions, we estimate the uncertainty region by performing a bootstrapping resampling-withreplacement MC analysis. The results give similar conclusions: we cannot discard the hypothesis that the two samples are drawn from the same distribution.

In Fig. 2 we report the plots resulting from the investigation of the SFR, specific SFR (sSFR), and metallicities of SLSN and LGRB hosts. The distributions of these properties are found to 

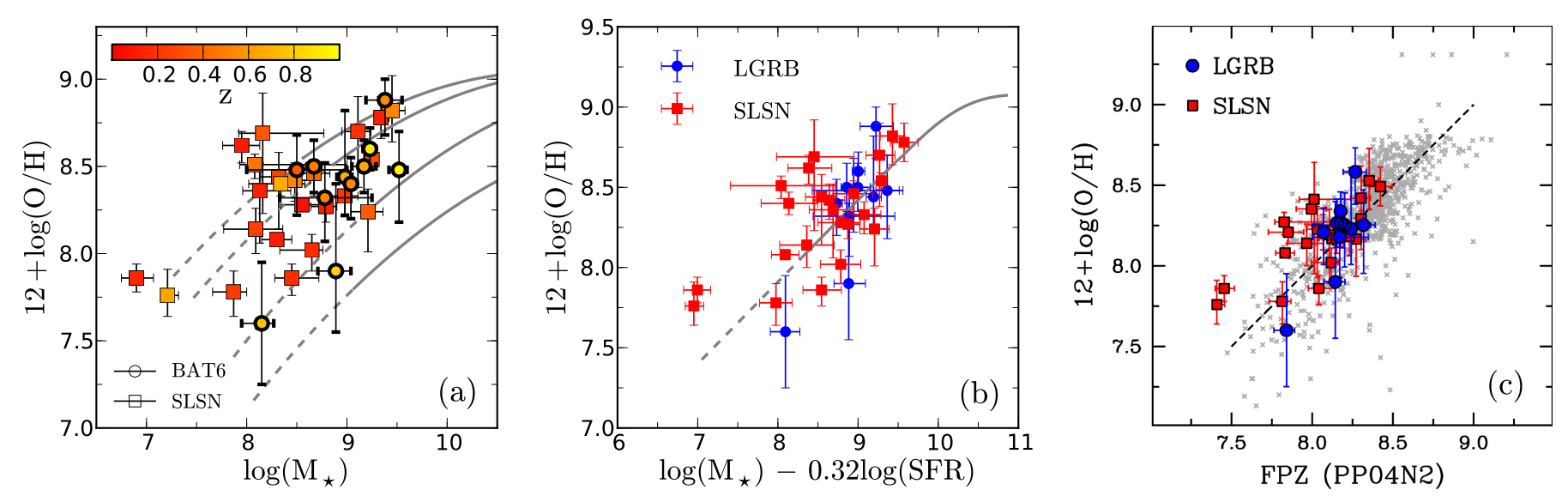

Fig. 3. Comparing the full LGRB and SLSN samples to relations that are followed by field star-forming galaxy populations. a) Mass-metallicity relation. Overplotted are the median relations of star-forming galaxies at redshifts (from top to bottom) $z=0.07,0.7,2.2$, and 3.5 (Mannucci et al. 2009). Data are colour-coded according to their redshift. b) Fundamental metallicity relation (line; Mannucci et al. 2010, 2011) for SLSNe and LGRBs. c) SLSN and LGRB host galaxies in the fundamental metallicity plane (FPZ) for low-mass galaxies (grey; Hunt et al. 2016). The plane plotted is done with [N II]/H $\alpha$ metallicity calibration (Pettini \& Pagel 2004). Dashed lines in a) and b) show the extrapolations of the relations towards lower masses, where the relations are poorly defined.

be very similar at this redshift range for both classes of objects. Two SLSN hosts lack a significant detection of emission lines in their spectra. Performing a similar test to the case of stellar masses (e.g. by adding artificial values to the distributions and looking for the point when the discrepancy between distributions becomes significant) it is clear that a large portion of a SLSN population would have to be missed in the surveys, which is unlikely, in order to make the SLSN host properties different from those of LGRB hosts.

\subsection{Relations between $M_{\star}, S F R$, and metallicity: comparison to star-forming galaxies}

We then check the behaviour of SLSN host galaxies with respect to the relations found between $M_{\star}$, SFR, and metallicity (massmetalicity, FMR Mannucci et al. 2010, and FPZ Hunt et al. 2016). Apart from the mass-metalicity relation (Tremonti et al. 2004; Mannucci et al. 2009), these relations are thought to be insensitive to redshift (at least at the redshift considered in this work) and so here we include all the LGRB hosts of the BAT6 $0.3<z<1$ sample, and we expand the SLSN sample by including the low-redshift $(z<0.3)$ SLSN hosts. This extended SLSN host galaxy sample includes all the objects (30) at $z<0.7$ studied by L14, A16, and P16, with exception of 2 objects that do not have NIR observations (see Table A. 2 for the full account of the SLSN sample). We then add the requirement of the determination of the metallicity. In the end, our samples are made of 11 LGRB hosts (of the 13 objects of the BAT6 sample at $0.3<z<1$; see also Japelj et al. 2016) and 24 SLSN hosts (of the 30 objects of the union of the L14, A16, and P16 samples at $z<0.7)$.

In Fig. 3a we plot the mass vs metallicity of the two samples at $z<1$. Focusing on the $0.3<z<0.7$ range, it seems that SLSN and LGRB hosts occupy the same region in the mass-metallicity relation and also agree on average with the median relations of field star-forming galaxies at the same redshifts. Approximately half of the low-redshift $(z<0.3)$ SLSN hosts are found to have significantly lower metallicities than the star-forming galaxies at the same stellar mass and redshift range.
LGRB host galaxies have been found to follow the fundamental metallicity relation (FMR), a two-dimensional surface defined by metallicities, SFRs, and stellar masses of the main-sequence star-forming galaxies (Mannucci et al. 2011; Japelj et al. 2016). In Fig. 3 we show that SLSN host galaxies also lie in the same plane with a similar dispersion to the LGRB hosts: the dispersion of LGRB and SLSN samples around the FMR relation is 0.22 dex and 0.26 dex, respectively. These values are larger than the typical dispersion of the FMR at $\log \left(M_{\star} / M_{\odot}\right) \gtrsim 9.4$, which can be attributed to the small number statistics and increasing intrinsic scatter of the FMR relation towards lower values of $\mu_{0.32}=\log M_{\star}-0.32 \log S F R$ (Mannucci et al. 2011). Both LGRB and SLSN hosts are predominantly low-mass galaxies with masses extending to the range at which the FMR is not well defined. Hunt et al. (2012, 2016) computed a scaling relation using stellar masses, SFRs, and metallicities toward low-mass starburst galaxies to build a fundamental metallicity plane (FPZ). We plot our LGRB and SLSN host galaxy samples in the FPZ and show that they lie within the region populated by other types of low-mass galaxies, with dispersion of 0.16 dex and 0.18 dex, respectively.

\section{Discussion}

Previous studies of SLSN host galaxies, from which our SLSN data have been compiled, reached different conclusions regarding the relative properties of SLSN and LGRB host galaxies. In order to get a more robust understanding of the two populations of host galaxies, (i) we used a complete sample of LGRBs for the comparison; (ii) we require the NIR photometry for the stellar mass determination; and (iii) we limited our analysis in the relevant cases to a narrower redshift range so as to minimize the impact of the evolution of the galaxy properties. Some of our results differ from the conclusions of previous studies.

L14 and L16 have found that, on average, SLSN hosts are much fainter than LGRB hosts. Because the faintness implies low masses and low metallicities, the result was inconsistent with the observed similarity between metallicity distributions of the two classes. On the contrary, we find that $M_{\mathrm{B}}$ and stellar mass distributions of the two classes are similar. The discrepancies of stellar masses, which are not statistically significant, could 


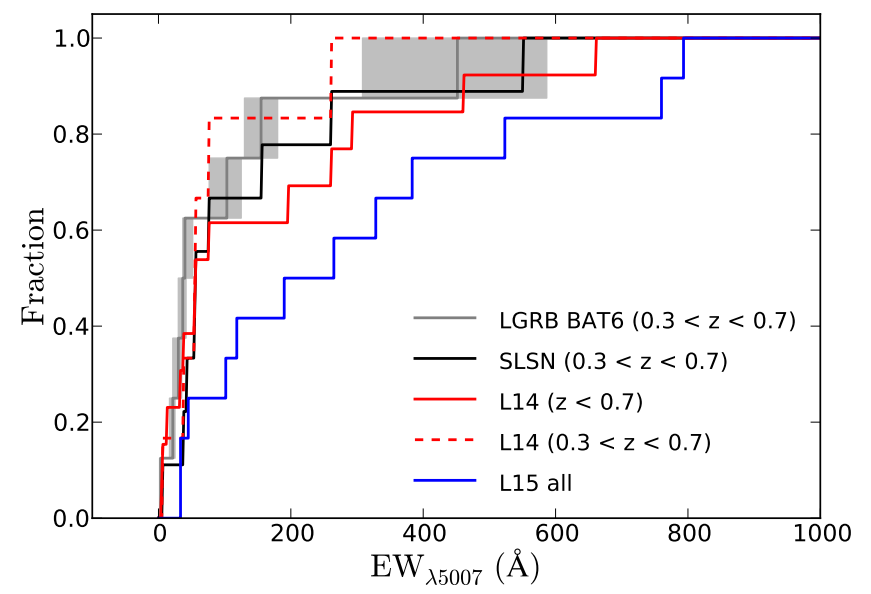

Fig. 4. Distributions of equivalent widths of the $[\mathrm{O}$ III $] \lambda 5007$ emission line. The distribution of $0.3<z<0.7$ LGRB sample values (grey) is compared to the SLSN sample (Table A.1; black) in the same redshift range. Furthermore, we plot distributions of SLSN samples from the literature, namely from L15 (blue) and L14 (red). Errors are not taken into account in the case of SLSN measurements as they are negligible.

be due to the bias of SLSN sample against dusty/luminous host galaxies (see L14, P16). We note that even if the slight preference for lower masses is intrinsic to the SLSN population, the effect is much smaller than claimed by some of the previous works. The similar nature of the LGRB and SLSN hosts is further corroborated by resolved imaging of the hosts. Using HST imaging, Lunnan et al. (2015) showed that SLSN and LGRB hosts have similar morphologies, effective galaxy radii, and SFR densities. They also found that SLSN are tracing UV-bright regions in their hosts, though the correspondence is not as strong as in the case of LGRBs (Fruchter et al. 2006).

SLSN hosts are notable for their strong emission lines. L15 compared the equivalent width distribution of the [O III $] \lambda 5007$ line for the LGRB and SLSN hosts and claimed that the fraction of extreme emission line galaxies (EELGs, e.g. Atek et al. 2011) with high emission line equivalent widths is much higher among SLSN hosts than among the LGRB hosts. Because the strength of the $\mathrm{EW}_{5007}$ diminishes with time after a starburst (e.g. Zackrisson et al. 2001; Inoue 2011), L15 concluded that the SLSNe represent host galaxies viewed shortly after a starburst event. Moreover, taking at face value the results by Zackrisson et al. (2001), the $\mathrm{EW}_{5007}$ is expected to strongly decrease after a few Myr corresponding roughly to the ZAMS lifetime of stars with masses of 50-60 $M_{\odot}$ (Meynet \& Maeder 2005). Therefore, assuming that both SLSN and LGRB progenitors are formed during an instantaneous starburst event, SLSN that originated by more massive, short-lived progenitors would be detected in galaxies with enhanced $\mathrm{EW}_{5007}$, while LGRBs would be related to the death of 20-30 $M_{\odot}$ (e.g. Thöne et al. 2008; Levesque et al. 2010a) and found in less extreme conditions.

However, the conclusions of L15 regarding the EW seem to be strongly affected by the domination of low- $z$ SLSN hosts in their sample, compared to the very small fraction of LGRBs at $z<0.3$ of their comparison sample. The effect is illustrated in Fig. 4, where we compare the distribution of $\mathrm{EW}_{5007}$ of the LGRB sample used in this paper ${ }^{2}$ to the SLSN distributions presented by L15 and L14. The sample of L15 is built

\footnotetext{
2 L15 shows the $\mathrm{EW}_{5007}$ distribution built for the TOUGH LGRB complete sample (Hjorth et al. 2012): the distribution is very similar to the one we built with our sample.
}

primarily of $z<0.3$ hosts and is indeed strikingly different from the LGRB distribution. However, the sample of L14, including much larger fraction of $z>0.3$ hosts, agrees much more closely with the LGRB values. Indeed, taking only the $z>0.3$ values from the L14 sample, the distribution is essentially the same as the one for LGRB hosts. Finally, we compare the values of the $\mathrm{EW}_{5007}$ for the LGRB hosts and SLSN sample defined in Table A.1 (e.g. in the $0.3<z<0.7$ redshift range; data provided by R. Lunnan and D. Perley, priv. comm.) and confirm that the two distributions are similar. Two SLSN without detected lines (PTF09cwl and PTF09atu) are faint (e.g. Perley et al. 2016b). However, the lack of detected emission lines suggests the lines are not very strong and should therefore not affect our conclusions. Furthermore, using the bootstrap method we confirm that the difference between our sample (high- $z$ ) and sample from Leloudas et al. (2015) (low-z) is not due to the small samples used in the comparison.

The previous finding of L15 can therefore mainly be attributed to the apparent redshift evolution of the $\mathrm{EW}_{5007}$ in SLSN hosts. According to Steidel et al. (2014) the ionization field, and therefore the strength of the emission lines, in star-forming galaxies becomes stronger with redshift, which is opposite to what we observe in SLSN hosts. A possible explanation is that the observed evolution is due to the size of galaxies in the sky: at lower redshifts the galaxies are resolved and a spectrograph slit can be placed at the positions pointed by SLSN explosions, while at higher redshifts the slit covers an entire galaxy and the obtained spectra represent an average over different nebular regions. This interpretation is supported by two hosts in L15 (hosts of SN1999as and PTF10qaf, the latter being SLSN-II), where spectra taken at different slit positions give different values of EW with the largest at the SLSN explosion cite. Owing to the lack of $z<0.3$ LGRBs in complete samples (Salvaterra et al. 2012; Hjorth et al. 2012; Perley et al. 2016a) we cannot make a more quantitative study of this issue. We note that other nebularline based galaxy characteristics (e.g. SFR) are affected by the same redshift-dependent effect. For example, low- $z$ SLSN samples show a strong preference for high SSFR with respect to the field star-forming galaxies (Perley et al. 2016b; Chen et al. 2016). It seems that at the redshift range studied in this paper, $0.3<z<0.7$, the fraction of SLSN hosts with very high SSFR decreases and is similar to the one observed in the LGRB host samples (Fig. 2). LGRB hosts at this redshift show only a slight preference for high sSFR galaxies with respect to the field starforming population, though due to low statistics involved in the studies the issue is still unclear (Japelj et al. 2016). In any case, if the spatial resolution is indeed the major cause of the seemingly different properties at low- $z$, it should not affect the comparison of LGRB and SLSN hosts at $z>0.3$ as their sizes and morphology are similar (e.g. Lunnan et al. 2015).

In the absence of any strong observable difference between LGRB and SLSN-I host galaxy environments, we are left with few new clues to improve our understanding of SLSN progenitors. The metallicities observed in SLSN hosts are preferentially low (e.g. see also Lunnan et al. 2014; Leloudas et al. 2015; Perley et al. 2016b; Chen et al. 2016), avoiding super solar metallicities. The similar distribution of SLSN and LGRB host galaxy metallicity that we find agrees with the results of Chen et al. (2016). Indeed, in Vergani et al. (2015) and Japelj et al. (2016) we find a similar preference for $Z<0.5 Z_{\odot}$ for LGRB host galaxies. This is in favour of the progenitor models requiring low metallicities, e.g. the magnetar model. It should be remembered that the similarity between LGRB and SLSN host galaxies does not necessarily imply that these two types of 
events have the same progenitors in general (but see the special case of ultra-long GRB 111209A and its accompanying SN; Greiner et al. 2015).

Finally, given a relatively small number of observed SLSN host galaxies, all current studies are affected by small number statistics. We note that even though we restricted our analysis to a narrow redshift range, excluding the $z<0.3$ SLSN population, the size of our sample is still of the same order as previously studied samples.

\section{Conclusions}

We compared the properties of the host galaxies of SLSNe to those of LGRBs with the aim of looking for similarities and possible differences between the two populations to try to understand the different results found in the literature on this topic and with the final goal of shedding some light on the progenitors of these two phenomena. In contrast to previous studies, we used the host galaxies of a complete sample of LGRB as comparison. Furthermore, we performed the comparison in a reduced redshift range, and carefully selected the published stellar masses of SLSN host galaxies.

Our results indicate that, at least within $0.3<z<0.7$, the properties of SLSN and LGRB host galaxies are similar in terms of their $M_{B}$, stellar masses, SFR, sSFR, and metallicities. We also show that the host galaxies of SLSN follow the FMR and FPZ relations as field star-forming galaxies. The discrepancies of the results of previous studies are due to the different (biased) comparison samples of LGRB host galaxies used, to the different stellar mass values used among all those studies, and to the comparison made over a wide/different redshift range for the two classes of explosions. We do not find evidence of the excess of high-EW SLSN hosts that were found by studies focused on low-z SLSN samples (Leloudas et al. 2015).

We note that larger samples are needed to improve these results, e.g. more high- $z$ SLSN and more low- $z$ LGRB host galaxies as well as a complete sample of SLSNe.

Acknowledgements. We thank the referee for the helpful comments that improved the paper. We thank R. Lunnan and D. Perley for providing EW measurements for the L14 and P16 samples of SLSN hosts. JJ acknowledges financial contribution from the grant PRIN MIUR 2012 201278X4FL 002 The Intergalactic Medium as a probe of the growth of cosmic structures.

\section{References}

Angus, C. R., Levan, A. J., Perley, D. A., et al. 2016, MNRAS, 458, 84 Atek, H., Siana, B., Scarlata, C., et al. 2011, ApJ, 743, 121 Barkat, Z., Rakavy, G., \& Sack, N. 1967, Phys. Rev. Lett., 18, 379 Berger, E., Chornock, R., Lunnan, R., et al. 2012, ApJ, 755, L29 Bloom, J. S., Perley, D. A., Li, W., et al. 2009, ApJ, 691, 723

Cano, Z., Johansson Andreas, K. G., \& Maeda, K. 2016, MNRAS, 457, 2761 Chabrier, G. 2003, PASP, 115, 763

Chen, T.-W., Smartt, S. J., Bresolin, F., et al. 2013, ApJ, 763, L28

Chen, T.-W., Smartt, S. J., Jerkstrand, A., et al. 2015, MNRAS, 452, 1567
Chen, T.-W., Smartt, S. J., Yates, R. M., et al. 2016, MNRAS, submitted [arXiv: 1605.04925]

Dessart, L., Hillier, D. J., Waldman, R., Livne, E., \& Blondin, S. 2012, MNRAS, 426, L76

Dexter, J. \& Kasen, D. 2013, ApJ, 772, 30

Fruchter, A. S., Levan, A. J., Strolger, L., et al. 2006, Nature, 441, 463 Gal-Yam, A. 2012, Science, 337, 927

Gal-Yam, A., Mazzali, P., Ofek, E. O., et al. 2009, Nature, 462, 624

Graham, J. F., \& Fruchter, A. S. 2013, ApJ, 774, 119

Greiner, J., Mazzali, P. A., Kann, D. A., et al. 2015, Nature, 523, 189

Hjorth, J., Malesani, D., Jakobsson, P., et al. 2012, ApJ, 756, 187

Hunt, L., Magrini, L., Galli, D., et al. 2012, MNRAS, 427, 906

Hunt, L., Dayal, P., Magrini, L., \& Ferrara, A. 2016, MNRAS, in press [arXiv: 1608.05417]

Inoue, A. K. 2011, MNRAS, 415, 2920

Inserra, C., Smartt, S. J., Jerkstrand, A., et al. 2013, ApJ, 770, 128

Inserra, C., Smartt, S. J., Gall, E. E. E., et al. 2016, ApJ, submitted [arXiv: 1604.01226]

Japelj, J., Vergani, S. D., Salvaterra, R., et al. 2016, A\&A, 590, A129

Kennicutt, Jr., R. C. 1998, ARA\&A, 36, 189

Krühler, T., Malesani, D., Fynbo, J. P. U., et al. 2015, A\&A, 581, A125

Kumar, P., \& Zhang, B. 2015, Phys. Rep., 561, 1

Langer, N., Norman, C. A., de Koter, A., et al. 2007, A\&A, 475, L19

Le Floc'h, E., Duc, P.-A., Mirabel, I. F., et al. 2003, A\&A, 400, 499

Leloudas, G., Schulze, S., Krühler, T., et al. 2015, MNRAS, 449, 917

Levesque, E. M., Kewley, L. J., Berger, E., \& Zahid, H. J. 2010a, AJ, 140, 1557

Levesque, E. M., Soderberg, A. M., Kewley, L. J., \& Berger, E. 2010b, ApJ, 725, 1337

Lunnan, R., Chornock, R., Berger, E., et al. 2014, ApJ, 787, 138

Lunnan, R., Chornock, R., Berger, E., et al. 2015, ApJ, 804, 90

Maiolino, R., Nagao, T., Grazian, A., et al. 2008, A\&A, 488, 463

Mannucci, F., Cresci, G., Maiolino, R., et al. 2009, MNRAS, 398, 1915

Mannucci, F., Cresci, G., Maiolino, R., Marconi, A., \& Gnerucci, A. 2010, MNRAS, 408, 2115

Mannucci, F., Salvaterra, R., \& Campisi, M. A. 2011, MNRAS, 414, 1263

Metzger, B. D., Margalit, B., Kasen, D., \& Quataert, E. 2015, MNRAS, 454, 3311

Meynet, G., \& Maeder, A. 2005, A\&A, 429, 581

Moriya, T. J., Blinnikov, S. I., Tominaga, N., et al. 2013, MNRAS, 428, 1020

Neill, J. D., Sullivan, M., Gal-Yam, A., et al. 2011, ApJ, 727, 15

Nicholl, M., Smartt, S. J., Jerkstrand, A., et al. 2013, Nature, 502, 346

Nicholl, M., Smartt, S. J., Jerkstrand, A., et al. 2015, MNRAS, 452, 3869

Perley, D. A., Krühler, T., Schulze, S., et al. 2016a, ApJ, 817, 7

Perley, D. A., Quimby, R., Yan, L., et al. 2016b, ApJ, submitted [arXiv: 1604.08207]

Perley, D. A., Tanvir, N. R., Hjorth, J., et al. 2016c, ApJ, 817, 8

Pettini, M., \& Pagel, B. E. J. 2004, MNRAS, 348, L59

Planck Collaboration XVI. 2014, A\&A, 571, A16

Quimby, R. M., Kulkarni, S. R., Kasliwal, M. M., et al. 2011, Nature, 474, 487

Salvaterra, R., Campana, S., Vergani, S. D., et al. 2012, ApJ, 749, 68

Savaglio, S., Glazebrook, K., \& Le Borgne, D. 2009, ApJ, 691, 182

Steidel, C. C., Rudie, G. C., Strom, A. L., et al. 2014, ApJ, 795, 165

Thöne, C. C., Fynbo, J. P. U., Östlin, G., et al. 2008, ApJ, 676, 1151

Thöne, C. C., de Ugarte Postigo, A., García-Benito, R., et al. 2015, MNRAS, 451, L65

Tremonti, C. A., Heckman, T. M., Kauffmann, G., et al. 2004, ApJ, 613, 898

Usov, V. V. 1992, Nature, 357, 472

Vergani, S. D., Salvaterra, R., Japelj, J., et al. 2015, A\&A, 581, A102

Vreeswijk, P. M., Savaglio, S., Gal-Yam, A., et al. 2014, ApJ, 797, 24

Woosley, S. E. 1993, ApJ, 405, 273

Woosley, S. E. 2010, ApJ, 719, L204

Woosley, S. E., \& Heger, A. 2006, ApJ, 637, 914

Yoon, S.-C., Langer, N., \& Norman, C. 2006, A\&A, 460, 199

Zackrisson, E., Bergvall, N., Olofsson, K., \& Siebert, A. 2001, A\&A, 375, 814 


\section{Appendix A: Additional tables}

Table A.1. Properties of the H-poor SLSN sample used in the paper for cumulative plots.

\begin{tabular}{lcccccc}
\hline \hline SLSN & Redshift & $\begin{array}{c}M_{\mathrm{B}} \\
(\mathrm{mag})\end{array}$ & $\begin{array}{c}\log M_{\star} \\
\left(M_{\odot}\right)\end{array}$ & $\begin{array}{c}S F R \\
\left(M_{\odot} \mathrm{yr}^{-1}\right)\end{array}$ & $\begin{array}{c}12+\log \left(\frac{\mathrm{O}}{\mathrm{H}}\right) \\
\mathrm{M} 08\end{array}$ & References \\
\hline MLS12110 & 0.303 & -19.39 & $9.25_{-0.06}^{+0.04}$ & $0.73 \pm 0.02$ & $8.54_{-0.05}^{+0.05}$ & 1,2 \\
PTF12mxx & 0.33 & -16.9 & $8.16_{-0.24}^{+0.61}$ & $0.11_{-0.02}^{0.04}$ & $8.69_{-0.46}^{+0.23}$ & 4,4 \\
PTF09cwl & 0.349 & -15.7 & $7.85_{-0.33}^{+0.23}$ & $<0.06$ & - & 4,4 \\
PTF10bjp & 0.359 & -18.8 & $9.21_{-0.15}^{+0.15}$ & $0.93_{-0.25}^{+0.28}$ & $8.24_{-0.23}^{+0.13}$ & 4,4 \\
SN2006oz & 0.396 & -16.96 & $8.67_{-0.04}^{+0.11}$ & $0.13 \pm 0.11$ & $8.46_{-0.13}^{+0.08}$ & 1,3 \\
PTF10vqv & 0.45 & -18.4 & $8.08_{-0.15}^{+0.92}$ & $1.36_{-0.36}^{+0.41}$ & $8.51_{-0.08}^{+0.06}$ & 4,4 \\
PTF09atu & 0.501 & -16.3 & $8.35_{-0.62}^{+0.33}$ & $<0.11$ & - & 4,4 \\
PS1-12bqf & 0.522 & -20.28 & $9.45_{-0.10}^{+0.13}$ & $1.05 \pm 0.55$ & $8.82_{-0.18}^{+0.20}$ & 2,2 \\
PS1-11ap & 0.524 & -18.83 & $8.48_{-0.12}^{+0.15}$ & $0.30 \pm 0.18$ & $8.42_{-0.12}^{+0.10}$ & 2,2 \\
PS1-10bzj & 0.650 & -17.90 & $7.21_{-0.05}^{+0.11}$ & $5.85 \pm 2.10$ & $7.76_{-0.12}^{+0.15}$ & 2,2 \\
PS1-12zn & 0.674 & -18.75 & $8.34_{-0.32}^{+0.24}$ & $4.50 \pm 2.00$ & $8.40_{-0.07}^{+0.07}$ & 2,2 \\
\hline
\end{tabular}

Notes. All objects have available NIR photometric observations and therefore reliably measured stellar masses. Reported are SLSN designation, redshift, absolute $B$-band magnitude, stellar mass, star formation rate, and nebular metallicity (in the M08 metallicity calibration). The last column lists the references from which the stellar masses and spectra were obtained, respectively. Masses and $M_{\mathrm{B}}$ were adopted directly from the referenced works, while SFR and metallicities were measured as described in Sect. 2.

References. (1) Angus et al. (2016); (2) Lunnan et al. (2014); (3) Leloudas et al. (2015); (4) Perley et al. (2016b). 
Table A.2. Properties of SLSN sample used to plot the relations in Fig. 3.

\begin{tabular}{|c|c|c|c|c|c|}
\hline SLSN & $\begin{array}{c}\text { Redshift } \\
\quad\left(M_{\odot}\right)\end{array}$ & $\begin{array}{c}\log M_{\star} \\
\left(M_{\odot} \mathrm{yr}^{-1}\right)\end{array}$ & $\begin{array}{l}S F R \\
\text { M08 }\end{array}$ & $12+\log \left(\frac{O}{H}\right)$ & References \\
\hline PTF11hrq & 0.057 & $8.55_{-0.21}^{+0.28}$ & $0.20_{-0.04}^{+0.04}$ & $8.28_{-0.03}^{+0.04}$ & 4,4 \\
\hline PTF10hgi & 0.098 & $7.95_{-0.19}^{+0.18}$ & $0.04 \pm 0.04$ & $8.62_{-0.10}^{+0.08}$ & 4,3 \\
\hline PTF12dam & 0.1078 & $8.30_{-0.15}^{+0.15}$ & $4.78_{-1.17}^{+0.96}$ & $8.08_{-0.03}^{+0.02}$ & 4,4 \\
\hline PTF10nmn & 0.123 & $8.79_{-0.15}^{+0.11}$ & $0.53_{-0.13}^{+0.12}$ & $8.27_{-0.09}^{+0.07}$ & 4,4 \\
\hline SN1999as & 0.127 & $9.34_{-0.00}^{+0.08}$ & $0.17 \pm 0.19$ & $8.78_{-0.12}^{+0.12}$ & 1,3 \\
\hline SN2007bi & 0.127 & $8.13_{-0.17}^{+0.31}$ & $0.02 \pm 0.01$ & $8.36_{-0.30}^{+0.16}$ & 1,3 \\
\hline SN2011ke & 0.143 & $6.90_{-0.15}^{+0.17}$ & $0.40 \pm 0.03$ & $7.86_{-0.08}^{+0.08}$ & 4,3 \\
\hline PTF10bfz & 0.175 & $8.65_{-0.32}^{+0.11}$ & $0.39_{-0.10}^{+0.10}$ & $8.02_{-0.12}^{+0.09}$ & 4,4 \\
\hline SN2012il & 0.169 & $8.45_{-0.17}^{+0.27}$ & $0.38 \pm 0.07$ & $7.86_{-0.10}^{+0.08}$ & 1,3 \\
\hline PTF10vwg & 0.19 & $8.25_{-0.59}^{+0.18}$ & $<0.07$ & - & 4,4 \\
\hline PTF11rks & 0.192 & $9.11_{-0.16}^{+0.13}$ & $0.29 \pm 0.13$ & $8.70_{-0.32}^{+0.20}$ & 4,3 \\
\hline PTF10aagc & 0.206 & $8.98_{-0.21}^{+0.13}$ & $0.47_{-0.16}^{+0.19}$ & $8.33_{-0.12}^{+0.08}$ & 4,4 \\
\hline SN2010gx & 0.230 & $7.87_{-0.21}^{+0.13}$ & $0.42 \pm 0.17$ & $7.78_{-0.14}^{+0.12}$ & 4,3 \\
\hline SN2011kf & 0.245 & $8.09_{-0.32}^{+0.27}$ & $0.15 \pm 0.05$ & $8.14_{-0.14}^{+0.12}$ & 1,3 \\
\hline PTF09cnd & 0.258 & $8.32_{-0.18}^{+0.15}$ & $0.23 \pm 0.06$ & $8.44_{-0.30}^{+0.14}$ & 4,3 \\
\hline SN2005ap & 0.283 & $8.46_{-0.15}^{+0.16}$ & - & - & $1,-$ \\
\hline PTF10uhf* & 0.289 & $10.6_{-0.2}^{+0.2}$ & $5_{-2}^{+2}$ & $8.98_{-0.01}^{+0.01}$ & 4,4 \\
\hline MLS12110 & 0.303 & $9.25_{-0.06}^{+0.04}$ & $0.73 \pm 0.02$ & $8.54_{-0.05}^{+0.05}$ & 1,2 \\
\hline PTF12mxx & 0.33 & $8.16_{-0.24}^{+0.61}$ & $0.11_{-0.02}^{0.04}$ & $8.69_{-0.46}^{+0.23}$ & 4,4 \\
\hline PTF09cwl & 0.349 & $7.85_{-0.33}^{+0.23}$ & $<0.06$ & - & 4,4 \\
\hline PTF10bjp & 0.359 & $9.21_{-0.17}^{+0.15}$ & $0.93_{-0.25}^{+0.28}$ & $8.24_{-0.23}^{+0.13}$ & 4,4 \\
\hline SN2006oz & 0.396 & $8.67_{-0.04}^{+0.11}$ & $0.13 \pm 0.11$ & $8.46_{-0.13}^{+0.08}$ & 1,3 \\
\hline PTF10vqv & 0.45 & $8.08_{-0.15}^{+0.92}$ & $1.36_{-0.36}^{+0.41}$ & $8.51_{-0.08}^{+0.06}$ & 4,4 \\
\hline PTF09atu & 0.501 & $8.35_{-0.62}^{+0.33}$ & $<0.11$ & - & 4,4 \\
\hline PS1-12bqf & 0.522 & $9.45_{-0.10}^{+0.13}$ & $1.05 \pm 0.55$ & $8.82_{-0.18}^{+0.20}$ & 2,2 \\
\hline PS1-11ap & 0.524 & $8.48_{-0.12}^{+0.15}$ & $0.30 \pm 0.18$ & $8.42_{-0.12}^{+0.10}$ & 2,2 \\
\hline PS1-10bzj & 0.650 & $7.21_{-0.05}^{+0.11}$ & $5.85 \pm 2.10$ & $7.76_{-0.12}^{+0.15}$ & 2,2 \\
\hline PS1-12zn & 0.674 & $8.34_{-0.32}^{+0.24}$ & $4.50 \pm 2.00$ & $8.40_{-0.07}^{+0.07}$ & 2,2 \\
\hline
\end{tabular}

Notes. The table lists all SLSN hosts complying with our selection criteria (Sect. 2), even though only hosts with detected emission lines and measured metallicities are used for the plotting. Reported are SLSN designation, redshift, stellar mass, star formation rate, and nebular metallicity (in the M08 metallicity calibration). The last column gives the references from which the stellar masses and spectra were obtained, respectively. Masses and $M_{\mathrm{B}}$ were adopted directly from the referenced works, while SFR and metallicities were measured as described in Sect. 2.

References. (1) Angus et al. (2016); (2) Lunnan et al. (2014); (3) Leloudas et al. (2015); (4) Perley et al. (2016b). ${ }^{(*)}$ Mass and SFR scaled by a factor of 4 to get the approximate values at the SLSN site, as suggested by (Perley et al. 2016b). 